\title{
INTERPRETIVE SUMMARIES, MARCH 2012
}

Invited review: Mastitis in dairy heifers: Nature of the disease, potential impact, prevention, and control. By De Vliegher et al., page 1025. Many dairy heifers freshen with mastitis. Some of the affected heifers remain prone to subclinical and clinical mastitis during first lactation. The impact of heifer mastitis is related to the form (clinical versus subclinical), the virulence of the causative pathogens, time of onset of infection relative to parturition, and immunity of the host. Prevention is based on optimizing hygiene, feeding and fly control, avoidance of cross-suckling in young stock, and improving animal comfort at parturition. Prepartum antibiotic treatment can be implemented only as a short-term measure to assist in the control of a significant heifer mastitis problem under supervision of the herd veterinarian.

Invited review: Role of physically effective fiber and estimation of dietary fiber adequacy in high-producing dairy cattle. By Zebeli et al., page 1041. Feeding adequate amounts of physically effective fiber (i.e., a measure of dietary fiber that takes into account both its chemical and physical features) and estimating dietary fiber adequacy are crucial aspects in the nutrition of high-producing dairy cattle. In this article, we provide an overview of the role, limitations, potentials, and practical recommendations in using the concept of physically effective fiber to estimate dietary fiber adequacy and formulate healthy diets for highproducing dairy cows.

Comparison of heat stability of goat milk subjected to ultra-high temperature and in-container sterilization. By Chen et al., page 105\%. Goat milk produced more sediment after ultra-high temperature (UHT) processing compared with in-container sterilization. When the ionic calcium was increased following addition of calcium chloride, sediment increased drastically after UHT treatment, but by much less following in-container sterilization. However, when ionic calcium was decreased following stabilizing salt addition, incontainer sterilized milk showed an increase in sediment, whereas UHT-treated milk first showed improved heat stability with less sediment. However, greater additions of stabilizing salt caused sediment to increase. Thus, heat stability mechanisms are different for these sterilization procedures, and poor heat stability can be found at both low and high ionic calcium concentrations.

A survey of the bacterial composition of kurut from Tibet using a culture-independent approach. By Liu et al., page 1064. Kurut (fermented yak milk) made by natural fermentation is a very important dairy food for local people in Tibet. It is necessary to comprehensively understand its bacterial composition for quality improvement and industrial production of kurut. A bacterial 16S rRNA gene clone library, including 460 clones, was constructed using total DNA extracted from 30 kurut samples. After screening by restriction fragment length polymorphism analysis, 56 operational taxonomic units (OTU) with unique restriction fragment length polymorphism patterns were obtained. Then, one representative sequence of every OTU was sequenced and phylogenetically analyzed. Both lactic acid bacteria and some unknown bacteria play crucial roles in kurut.

Identification of lactic acid bacteria in traditional fermented yak milk and evaluation of their application in fermented milk products. By Cai et al., page 1073. The natural population of lactic acid bacteria in Xueo, a traditional fermented yak milk, was studied based on phenotypic properties, amplified ribosomal DNA restriction analysis, and sequence analyses of $16 \mathrm{~S}$ rDNA and atpA. Enterococcus durans, Lactobacillus fermentum, and Lactobacillus paracasei were the dominant species in Xueo. Enterococcus durans contributes to the viscous texture of Xueo, and Lactobacillus species mainly produce acidity and contribute to the product's unique flavor. We recommend mixing the selected strains as starters to develop Xueo-flavored yogurt.

The effects of information on willingness to pay for animal welfare in dairy production: Application of nonhypothetical valuation mechanisms. By Elbakidze and Nayga, page 1099. Intensification of agricultural production, including dairy production, has contributed significantly to availability of relatively inexpensive food. However, along with enhanced productivity, intensification of agricultural production, dairy industry included, brought forth production-related ethical concerns such as sustainability, eco-footprint, and animal welfare. It is important to address such issues not only from a supply perspective but also from a demand perspective. Consumer willingness to pay for less intensively produced and potentially more costly animal products is an important factor of interest to livestock industry and policy makers. Using experimental techniques, we detected limited willingness to pay a premium price for humane animal care-labeled ice cream and we found no willingness to pay a premium price for humane animal care-labeled cheese.

$\beta$-Casein hydrolysate generated by the cell envelope-associated proteinase of Lactobacillus delbrueckii subspecies lactis CRL 581 protects 
against trinitrobenzene sulfonic acid-induced colitis in mice. By Espeche Turbay et al., page 1108. Lactobacillus delbrueckii ssp. lactis CRL 581, a thermophilic lactic acid bacterium used as a starter culture for the manufacture of several fermented dairy products, can release bioactive health beneficial peptides from milk proteins. The $\alpha$ - and $\beta$-casein hydrolysates generated by this strain exhibited antimutagenic activity in vitro. In addition, the $\beta$-casein hydrolysate had an antiinflammatory effect on colitis in mice. Our findings open up opportunities for developing novel functional foods.

Effects of small-diameter silver nanoparticles on microbial load in cow milk. By Yildiz and Pala, page 1119. Silver nanoparticles can now be used to coat various surfaces inexpensively. Antibacterial apparatuses or surfaces prevent microbial growth in milk, aiding the cooling chain and thermal processing of milk. This research investigated effects of time, temperature, and accelerating voltage of small diameter silver nanoparticle-coated braids on microbial load in milk and their effectiveness on different microorganisms. The particles were effective across times and temperatures, but lost their effectiveness in $10 \mathrm{~h}$, indicating that milk can later be processed to dairy products requiring use of bacterial cultures. The nanoparticles were effective on coliform bacteria, Escherichia coli, and Staphylococcus aureus but not on yeasts and molds.

Diacetyl levels and volatile profiles of commercial starter distillates and selected dairy foods. By Rincon-Delgadillo et al., page 1128. Diacetyl is a common volatile present in many fermented dairy foods as a product of citrate metabolism. As a means of increasing the buttery aroma imparted by diacetyl, food manufacturers may add a GRAS (generally regarded as safe)-listed ingredient referred to as starter distillate. Products of fermentation and distillation, starter distillates contain many fermentation volatiles, but are primarily defined by the presence of diacetyl. Diacetyl has recently come under scrutiny for its potential toxicological significance. This paper details the presence of diacetyl and other volatiles in starter distillate products and foods containing starter distillates.

Determination of sialic acids in infant formula by chromatographic methods: A comparison of high-performance anion-exchange chromatography with pulsed amperometric detection and ultra-high-performance liquid chromatography methods. By Hurum and Rohrer, page 1152. Infant formulas, which are often based on bovine milk, may be supplemented to more closely match human milk. One of these supplements is whey protein, which adds sialic acids in the form of glycoproteins to the formula. These sialic acids may be determined in the samples by 2 independent chromatographic methods: ultrahigh-performance liquid chromatography or high-performance anion-exchange chromatography with pulsed amperometric detection. Two assays for sialic acids in infant formula were developed and are compared in this article.

Short communication: Examination of milk filters by real-time PCR as a herd-level indicator of Mycobacterium avium subspecies paratuberculosis presence in dairy herds. By Slana et al., page 1162. This study describes the real-time PCR method for the detection of Mycobacterium avium ssp. paratuberculosis in milk filters as the potential tool to assess paratuberculosis on the herd level. This fast and cost-effective method may assist in establishing the infection status of a herd and in choosing a suitable control strategy.

Short communication: Detection of Shiga toxinproducing Escherichia coli (STEC) in healthy cattle and pigs in Lima, Peru. By Rivera et al., page 1166. The aim of this study was to determine the prevalence of Shiga toxin-producing Escherichia coli (STEC) in farm animals in Lima, Peru. Stool samples were obtained from 114 cattle and 112 pigs from 10 and 4 farms, respectively. Shiga toxin-producing Escherichia coli were identified by PCR in 16 cattle (14\%) and in no pigs. All STEC strains had the Shiga toxin-producing gene stx1; only one had both stx1 and stx2. To our knowledge, this is the first study on the prevalence of STEC in farms animals in Peru using molecular methods.

The effect of premilking udder preparation on Holstein cows milked 3 times daily. By Watters et al., page 1170. A commercial dairy was used to determine the effects of preparation-lag routines on cows milked 3 times daily. Milking unit on-time for early- to mid-lactation cows did not differ for any treatments compared with dip plus forestrip and $90 \mathrm{~s}$ of lag time. However, lag times of $\leq 60 \mathrm{~s}$ compared with dip plus forestrip and $90 \mathrm{~s}$ of lag time increased unit on-time for late-lactation cows. Dairy producers have flexibility when choosing their premilking routine.

A reduction in milking frequency and feed allowance improves dairy cow immune status. By O'Driscoll et al., page 117\%. Cows experience an unavoidable period of immunosuppression during the peripartum period. This study showed how this can be ameliorated somewhat by using management strategies that reduce milk yield. Milking frequency and dry matter allowance had different effects on leukocyte differential; however, cows exposed to treatments that could be expected to reduce milk yield (i.e., low dry matter allowance and once-daily milking) expressed differential 
cell count patterns indicative of slightly improved immune system functioning, as well as reduced milk yield and mobilization of body reserves. Thus, utilization of strategies to reduce milk yield at the start of lactation could reduce stress and alter cow welfare and health.

Characterization of bovine glucose transporter 1 kinetics and substrate specificities in Xenopus oocytes. By Bentley et al., page 1188. Glucose uptake in the mammary gland is a rate-limiting step in milk production. Glucose transporter 1 (GLUT1) is the major glucose transporter in bovine mammary gland and plays a critical role in mammary glucose uptake. In this study, we characterized bovine GLUT1 transport kinetics and substrate specificities in Xenopus oocytes. Our study provides insight into the function of GLUT1 in glucose uptake in bovine mammary gland.

Effect of feed restriction on metabolites in cerebrospinal fluid and plasma of dairy cows. By Laeger et al., page 1198. Metabolites act as hunger or satiety signals in the brain and play an important role in the control of feed intake. These substances are also components of the cerebrospinal fluid (CSF) that surrounds the hypothalamus and brainstem, 2 major centers of feed intake regulation. The aim of this study was to elucidate whether CSF and plasma metabolites change in response to different feeding levels. The amino acids serine, threonine, and tyrosine were decreased only in CSF after feed restriction, which make these 3 metabolites putative anorexigenic signals for control of feed intake in cows.

Antimicrobial use on Canadian dairy farms. By Saini et al., page 1209. The present study quantified antimicrobial use on 89 Canadian dairy farms to determine the effect of antimicrobial use on antimicrobial resistance. Producers deposited empty drug containers in specially provided receptacles; these containers were tallied on drug tally sheets. $\beta$-Lactams (third-generation cephalosporins, penicillins), penicillin combinations containing colistin, tetracyclines, trimethoprimsulfonamide combinations, and lincosamides were most commonly used, whereas fluoroquinolones were rarely used on Canadian dairy farms. Milk production and overall antimicrobial drug use were positively correlated. Except for third-generation cephalosporins and tetracyclines, variation in overall antimicrobial drug use rate across 4 regions was not evident.

Effect of conjugated linoleic acid supplementation on body composition, body fat mobilization, protein accretion and energy utilization in early lactation dairy cows. By Danicke et al., page 1222. Conjugated linoleic acids (CLA) alter body composition by changes in nutrient partitioning in different mammals. The present study investigated the occurrence of these effects during CLA feeding in dairy cows. The naturally occurring mobilization of body mass (fat and protein) during the first $42 \mathrm{~d}$ in milk tended to be decelerated by CLA supplementation. The continuous CLA feeding until $105 \mathrm{~d}$ in milk increased protein accretion. These effects, in addition to a slightly decreased heat production and an unchanged milk energy output in the CLA groups, suggested enhanced utilization of metabolizable energy during early lactation.

Associations of subclinical hypocalcemia at calving with milk yield, and feeding, drinking, and standing behaviors around parturition in Holstein cows. By Jawor et al., page 1240. Fifteen Holstein dairy cows classified as having subclinical hypocalcemia within $24 \mathrm{~h}$ following calving were matched with 15 cows of normal calcium status, controlling for parity and other disease incidence. Differences in behavior before and after calving and milk yield between the 2 groups were evaluated. Cows with subclinical hypocalcemia produced nearly $6 \mathrm{~kg}$ more milk per day during wk 2, 3, and 4 after calving; however, feed intake was only slightly increased (wk 2 only) and water intake was not different between groups postpartum, although the frequency of visits to the water and feed bins decreased after calving. The subclinical hypocalcemia cows had greater dry matter intake before calving. Standing time was $2.6 \mathrm{~h}$ greater for cows diagnosed with subclinical hypocalcemia on the day before calving but these animals spent $2.7 \mathrm{~h}$ less time standing on the day after calving. Subclinical hypocalcemia is associated with changes in behavior.

Gluconeogenic enzymes are differentially regulated by fatty acid cocktails in Madin-Darby bovine kidney cells. By White et al., page 1249. Glucose production via gluconeogenesis is critical to lactation in dairy cows during transition. Changes in expression of enzymes that regulate gluconeogenesis have been observed at calving and during lactation, suggesting regulation by physiological cues during these periods. The effect of fatty acids on messenger RNA expression was examined in cells exposed to fatty acid cocktails that mimicked circulating fatty acid profiles before and at calving. Fatty acid exposure regulated key gluconeogenic enzymes differentially in a concentration- and profile-dependent manner. This suggests a likely role for previously characterized changes in circulating fatty acids to regulate gluconeogenic enzymes.

The association between metabolic parameters and oocyte quality early and late postpartum in Holstein dairy cows. By Matoba et al., page 125\%. Infertility in dairy cattle is a multifactorial problem that may be linked to follicle development and the quality of 
the ovulated oocyte, to sperm transport and fertilization, to the reproductive tract environment, or to a combination of these factors. Here, using transvaginal oocyte recovery techniques coupled with in vitro fertilization, we attempted to examine the effect of duration postpartum, during which time cows typically undergo negative energy balance, on oocyte quality assessed in terms of morphology and development. Despite differences in metabolic profiles during the postpartum period, we found no evidence for an effect on oocyte quality.

A linear programming model to optimize diets in environmental policy scenarios. By Moraes et al., page 126\%. An optimization model was developed to formulate minimum cost diets when environmental policies exist and to study effects of these policies on diet formulation, methane emissions, and nitrogen and mineral excretion. Two types of policies on methane emissions were incorporated in the model structure: taxes and reduction of predetermined amounts of methane emissions. The model was effective in formulating minimum cost diets for dairy cattle and examining effects of each type of policy on diet formulation, diet composition, and prediction of methane emissions and nitrogen and mineral excretion.

Manageable risk factors associated with the lactational incidence, elimination, and prevalence of Staphylococcus aureus intramammary infections in dairy cows. By Dufour et al., page 1283. A longitudinal cohort study was carried out to identify management practices that can be used on dairy farms to prevent acquisition or increase elimination of intramammary infections caused by Staphylococcus aureus. The results indicate that reducing acquisition of new infections can have a greater effect on prevalence of infection than increasing elimination of existing infections. Numerous practices, mainly related to milking procedures, may be used by dairy producers to prevent acquisition of infections.

The association of serum metabolites in the transition period with milk production and early-lactation reproductive performance. By Chapinal et al., page 1301. A total of 2,365 dairy cows from 55 freestall herds across North America had blood samples collected weekly, from 1 wk before through 3 wk after calving, to investigate the effect of serum nonesterified fatty acids (NEFA), $\beta$-hydroxybutyrate (BHBA), and calcium on milk yield and pregnancy at the first artificial insemination. Elevated serum NEFA and BHBA and low serum calcium concentrations around parturition were associated with milk loss in early lactation. Low serum calcium concentrations were associated with decreased pregnancy at first artificial insemination.
Genetic merit for fertility traits in Holstein cows: I. Production characteristics and reproductive efficiency of Holstein cows in a pasturebased system. By Cummins et al., page 1310. To elucidate the underlying physiological basis of declining reproductive performance, the current study compared the phenotypic performance of cows with divergent genetic merit for fertility traits but with similar genetic merit for milk production traits. Superior genetic merit for fertility traits was associated with improved fertility performance, increased circulating concentrations of insulin-like growth factor-I, and greater body condition score. These results highlight the important contribution of genetic merit for fertility to phenotypic reproductive performance, which may not necessarily be to the detriment of milk production.

Effects of monensin on metabolic parameters, feeding behavior, and productivity of transition dairy cows. By Mullins et al., page 1323. The effects of dietary monensin were evaluated in 32 Holstein cows for $3 \mathrm{wk}$ before and after calving. Monensin increased meal frequency and minimized ruminal $\mathrm{pH}$ variance on the first day after calving. Monensin treatment also significantly increased abundance of carnitine palmitoyl transferase 1a, a mediator of liver fatty acid oxidation. Consistent with previous results, monensin significantly decreased plasma ketone concentrations, but did not alter concentrations of the other blood parameters measured. No significant effects on milk production or disease incidence were detected.

The effect of stocking rate and calving date on reproductive performance, body state, and metabolic and health parameters of HolsteinFriesian dairy cows. By McCarthy et al., page 133\%. Following abolition of the European Union's milk quota, increased milk production is likely to be realized in Ireland through more intensive grass-based production systems characterized by increased stocking rates. Although resulting in increased requirement for reproductive intervention, increased stocking rates are unlikely to have a deleterious effect on pregnancy rates or health status of the Irish dairy herd. Furthermore, the presence of a strain of Holstein-Friesian by stocking rate interaction indicates that selection for a betterconditioned New Zealand strain may be more appropriate for grass-based dairy systems with an increased stocking rate.

A novel diagnostic technique to determine uterine health of Holstein cows at 35 days postpartum. By Machado et al., page 1349. Uterine postpartum diseases in dairy cattle result in impaired reproductive performance and economic losses. This study evaluated the use of uterine lavage sample (35 d postpartum) optical density (ULSOD; measured at 620 
$\mathrm{nm}$ ) as a diagnostic tool for clinical endometritis and its association with metritis and retained placenta. Parity, metritis, and intrauterine Arcanobacterium pyogenes infection were significantly associated with ULSOD. Cows with ULSOD $\leq 0.058$ were 1.21 times more likely to conceive than cows with ULSOD $>0.058$ (positive for clinical endometritis).

Milk production and somatic cell counts: A cow-level analysis. By Hand et al., page 1358. The economic impact of milk loss due to mastitis is well known throughout the dairy industry. Milk loss is quantified by its relationship to somatic cell count either within a 24-h period or across lactations.

Short communication: Prepartum plasma insulin-like growth factor-I concentrations based on day of insemination are lower in cows developing postpartum diseases. By Piechotta et al., page 136\%. Early detection of dairy cows predisposed to postpartum production diseases is crucial. Cows that developed postpartum ketosis had increased blood nonesterified fatty acids (NEFA) concentrations before calving, whereas those with any postpartum disease had significantly lower insulin-like growth factor-I (IGF-I) concentrations 242 to $248 \mathrm{~d}$ after artificial insemination. Blood sampling is recommended relative to day of insemination in the prepartum period to assess NEFA and IGF-I, due to ease of implementation at the farm level.

Short communication: Presence of G proteincoupled receptor 43 in rumen epithelium but not in the islets of Langerhans in cattle. By Wang et al., page 1371. The mechanisms by which volatile fatty acids (VFA) stimulate rumen epithelial growth and pancreatic secretion of insulin and glucagon in cattle are unclear. It was recently discovered that VFA can bind to G protein-coupled receptor 43 (GPR43). This study demonstrated that GPR43-immunoreactive cells were present in the epithelium but not in the other layers of rumen wall and that GPR43-immunoreactive cells were not detected in the pancreas in cattle. These results support a potential role of GPR43 in directly mediating the effect of VFA on rumen epithelial growth, but not that on pancreatic secretion of insulin and glucagon in cattle.

Characterization of the disappearance and formation of biohydrogenation intermediates during incubations of linoleic acid with rumen fluid in vitro. By Honkanen et al., page 1376. Dietary unsaturated fatty acids are extensively hydrogenated in the rumen, but the kinetics of these reactions and formation of minor intermediates are not well characterized. Incubations with ruminal fluid indicated that the rate of linoleic acid (LeA) disappearance was dependent on the amount of added substrate. The study also confirmed that reduction of trans 18:1 to 18:0 was rate limiting and that hydrogenation of LeA resulted in the formation of numerous intermediates. Accumulation of several unusual 18:2 isomers, including cis-7,cis-12 18:2 and cis-8,cis-12 18:2, indicated that biohydrogenation of LeA also proceeds via mechanisms other than isomerization of the cis-12 double bond.

Effect of time duration of ruminal urea infusions on ruminal ammonia concentrations and portaldrained visceral extraction of arterial urea- $\mathrm{N}$ in lactating Holstein cows. By Røjen and Kristensen, page 1395. This study investigated urea-N recycling as a mechanism by which dairy cows can buffer infrequent nitrogen supplementation. Cows were fed a low- $\mathrm{N}$ basal diet combined with either continuous or $6 \mathrm{~h} / \mathrm{d}$ infusion of the same total amount of urea per kilogram of dry matter intake. We observed a carry-over effect for arterial urea- $\mathrm{N}$ concentration, but not for ruminal ammonia concentration. Cows did not increase urea-N transport from blood to the gastrointestinal tract during periods with low ruminal ammonia concentration. Urea- $\mathrm{N}$ transport is apparently not effective in buffering infrequent $\mathrm{N}$ supply in dairy cows.

Prediction of immunoglobulin G content in bovine colostrum by near-infrared spectroscopy. By Rivero et al., page 1410. Immunoglobulin G (IgG) content is critical to bovine colostrum quality. A fast, reliable method to evaluate colostral IgG concentration could allow farmers to identify superior colostrum. This work evaluated near infrared spectroscopy (NIRS) as a rapid method to predict the IgG content of bovine colostrum. Spectra of colostrum from 2 Holstein dairy farms (157 cows) were obtained and regression models developed for IgG content as measured by radial immunodiffusion. With appropriate mathematical treatment of the spectral data, reliable predictions $\left(\mathrm{R}^{2}=0.94\right)$ were obtained for IgG content and hence, colostrum quality.

A fibrolytic enzyme additive for lactating Holstein cow diets: Ruminal fermentation, rumen microbial populations, and enteric methane emissions. By Chung et al., page 1419. Effects of an exogenous fibrolytic enzyme additive on ruminal fermentation, rumen microbial populations, and enteric methane $\left(\mathrm{CH}_{4}\right)$ emissions were measured in 9 ruminally cannulated, lactating Holstein cows. The enzyme did not alter ruminal volatile fatty acid or $\mathrm{pH}$ profile, but increasing the level of enzyme in the diet linearly increased population densities of certain bacterial species and enteric $\mathrm{CH}_{4}$ emissions. More research using longer term studies is needed for this exogenous fibrolytic en- 
zyme additive to establish whether the observed shift in ruminal bacterial communities improves feed conversion efficiency of dairy cows.

Effect of physical damage to ears of corn before harvest and treatment with various additives on the concentration of mycotoxins, silage fermentation, and aerobic stability of corn silage. By Teller et al., page 1428. Damaging ears of corn before harvest resulted in plants with higher levels of selected mycotoxins but lower concentrations of starch at harvest. Addition of a microbial inoculant or potassium sorbate at ensiling had mixed effects on concentrations of mycotoxins after ensiling but consistently improved the aerobic stability of the silages. Corn silage that has been subject to severe physical damage prior to harvest (e.g., bird or insect damage or hail) should be tested for mycotoxins prior to feeding.

An unprotected conjugated linoleic acid supplement decreases milk production and secretion of milk components in grazing dairy ewes. By Oliveira et al., page 143\%. An unprotected conjugated linoleic acid supplement was shown to reduce fat content and yield in dairy ewes. However, the high dose also reduced milk yield and secretion of milk protein and lactose. These undesirable effects appear to be related to off-target effects from the high conjugated linoleic acid dose.

Effect of dietary fat blend and monensin supplementation on dairy cattle performance, milk fatty acid profiles, and milk fat depression. $\mathrm{By} \mathrm{He}$ et al., page 144\%. Diet fatty acid profiles may determine the extent of milk fat depression experienced when these fatty acids are accessible to rumen microbial activity. We evaluated the effect of increasing dietary oleic and linoleic acid, both independently and together, on milk fatty acid yields. Milk fat depression was associated with both dietary oleic and linoleic acid, but oleic was less potent than linoleic. Interactions with monensin were minimal and monensin had no significant effect on milk fat yield, but monensin affected milk fatty acid profiles in a way that suggested altered rumen biohydrogenation of dietary fatty acids.

Measuring residual feed intake in dairy heifers fed an alfafa (Medicago sativa) cube diet. By Waghorn et al., page 1462. A trial was undertaken to screen 1,052 Holstein-Friesian heifers aged 6 to 9 mo to identify individuals that used feed more or less efficiently for growth (residual feed intake). They were fed in pens, and intakes of alfalfa cubes were measured for 49 d. Forage-based cubes were fed because New Zealand dairy cows graze mostly pasture as the diet for milk production. The $10 \%$ most efficient animals ate
$21.7 \%$ less dry matter than the $10 \%$ least efficient, for similar rates of body weight gain.

Effect of corn silage harvest maturity and concentrate type on milk fatty acid composition of dairy cows. By Khan et al., page 1472. This study describes the effects of feeding corn silages, ensiled at different maturities, in combination with a high or low degradable carbohydrate concentrate on nutrient intake, milk production, and composition of milk and milk fat in the early lactating dairy cows. Corn harvest maturity, dry matter content of 300 to $420 \mathrm{~g} / \mathrm{kg}$ of fresh weight, decreased the content of C18:3n-3 and total n-3 and increased the n-6:n-3 ratio in milk fat of dairy cows. Between the concentrates, addition of the highly degradable carbohydrate concentrate increased the content of all C18:1 trans isomers, C18:2 cis-9,trans-11, C18:2 trans-10,cis-12, and total trans FA in milk fat.

Comparison of effects of dietary coconut oil and animal fat blend on lactational performance of Holstein cows fed a high-starch diet. By Hollmann and Beede, page 1484. We compared lactational performance in Holstein cows fed diets supplemented with $5 \%$ coconut oil (dry basis) as a source of medium-chain fatty acids to diets with either an animal fat blend as a source of long-chain fatty acids or no added fats in a high-starch diet. Overall, feeding fat lowered feed intake and solids-corrected milk yield. Five percent coconut oil reduced feed consumption quickly and dramatically and was discontinued. A mixture of animal fat blend and coconut oil (2.5\%:2.5\%, dry basis) reduced digestibility of neutral detergent fiber. Dietary coconut oil does not appear to be a useful feed ingredient for lactating dairy cows.

Establishment of ruminal enzyme activities and fermentation capacity in dairy calves from birth through weaning. By Rey et al., page 1500. The aim of this study was to characterize the establishment of fermentation parameters in dairy calves. At d 1 after birth, the ruminal redox potential value was positive and xylanase or amylase activities and volatile fatty acids were not detected. At d 2 after birth, the negative ruminal redox potential, the presence of xylanase and amylase activities, and the detection of fermentation end products (volatile fatty acids and ammonia nitrogen) reflected fermentative activity. These activities varied from d 2 to 1 mo after birth and then stabilized.

Comparison and improvements of different Bayesian procedures to integrate external information into genetic evaluations. By Vandenplas and Gengler, page 1513. One of the most important theoretical assumptions of methods used to assess genetic values is that all available information has 
to be considered simultaneously to obtain unbiased estimates. However, this assumption is often not adequately fulfilled, especially in terms of genomic selection. Integration of external information into genetic evaluations by a Bayesian procedure can partially resolve this problem. Integrated evaluations by avoiding double counting among animals were highly similar to a joint evaluation.

Food intake, milk production and tissue changes of Holstein-Friesian and Jersey $\times$ HolsteinFriesian dairy cows within a medium-input grazing system and a high-input total confinement system. By Vance et al., page 152\%. A decline in functional traits (e.g., fertility and health) within the Holstein-Friesian breed has prompted renewed interest in crossbreeding. The current experiment examined the performance of Holstein-Friesian and Jersey $\times$ Holstein-Friesian dairy cows within 2 contrasting milk production systems (grazing and confinement). Crossbred cows produced milk with a higher fat and protein content than Holstein cows; however, solids-corrected milk yield was unaffected by genotype. Holstein cows produced a greater milk yield response to concentrate supplementation than crossbred cows and tended towards a greater solids-corrected milk yield response.

Genetic analysis of pathogen-specific clinical mastitis in Norwegian Red cows. By Haugaard et al., page 1545. Records from 234,088 first-lactation daughters of 1,656 Norwegian Red sires were used for genetic analyses of pathogen-specific clinical mastitis. The point estimates of heritability of liability were $0.06,0.04,0.02$, and 0.03 for unspecific clinical mastitis, Staphylococcus aureus, Streptococcus dysgalactiae, and Escherichia coli clinical mastitis, respectively. The genetic correlation among the 4 traits ranged from 0.75 to 0.87 . Genetic correlations $<1$ indicate that clinical mastitis caused by different pathogens should be considered as partly different traits.

Use of the Illumina Bovine3K BeadChip in dairy genomic evaluation. By Wiggans et al., page 1552. Genomic evaluations using genotypes from the Illumina Bovine3K BeadChip became available in September 2010. Of the 2,900 single nucleotide polymorphisms (SNP) on the chip, 2,683 were found useful for genomic evaluation. Genotypes for SNP not on the chip were imputed. For animals genotyped with both $3 \mathrm{~K}$ and 50K BeadChips, $96.3 \%$ of genotypes from the $3 \mathrm{~K}$ BeadChip matched those from the 50K BeadChip after imputation. The correlation of evaluations from the 2 chips was 0.959 for Holstein and 0.963 for Jersey. The 3K BeadChip supports evaluations of adequate accuracy and its low cost has greatly increased genotyping of females.
Short communication: Interactions of milk, fat, and protein yield genotypes with herd feeding characteristics. By Dekleva et al., page 1559. Response to selection for milk, fat, and protein yields were compared in 11 tie-stall herds that varied in their nutrition management practices. Results suggested an interaction of genotype and feeding management system, with selection responses depressed in herds that had a large proportion of cows without feed available at the end of $24 \mathrm{~h}$ and in herds that fed a diet low in energy. Larger and fatter cows were more negatively affected by limited feed availability than smaller cows.

Short communication: Association of an OLR1 polymorphism with milk production traits in the Israeli Holstein population. By Wang et al., page 1565 . The objective of this study was to analyze the association between variants of the oxidized lowdensity lipoprotein (lectin-like) receptor 1 (OLR1) gene and milk production and health traits in the Israeli Holstein cattle population. A single nucleotide polymorphism in the 3 -untranslated region of the gene showed significant associations with milk protein percentage and somatic cell score.

Evaluation of various selective media for the detection of Pseudomonas species in pasteurized milk. By Van Tassell et al., page 1568. The objective of this study was to evaluate various gram-negative selective media for their abilities to quantitatively detect Pseudomonas spp. in fluid milk. The media tested include violet red bile agar, crystal violet tetrazolium agar, MacConkey's agar, and 3M Coliform Petrifilm. The Pseudomonas strains used in this study were all isolated from milk and were chosen to represent a diversity of species found in dairy products. Crystal violet tetrazolium agar outperformed all other selective media tested and therefore can be used to sensitively detect postpasteurization contamination of fluid milk by Pseudomonas spp.

Lactating dairy cows adapt quickly to being milked by an automatic milking system. By Jacobs and Siegford, page 1575. Little is known about how quickly cows with previous parlor experience adapt to being milked by an automatic milking system (AMS). The objective was to assess adaptation to milking in an AMS by measuring milk yield and behaviors associated with the stress response over time. Cows produced more milk and vocalized and eliminated less in the milking stall by d 1; however, step-kick responses varied over time. According to most measures, cows adapted to milking in the AMS by $\mathrm{d} 1$. 